\title{
MALT Lymphoma of the Urinary Bladder Shows a Dramatic Female Predominance, Uneven Geographic Distribution, and Possible Infectious Etiology
}

This article was published in the following Dove Press journal:

Research and Reports in Urology

\author{
Kirill A Lyapichev $\mathbb{1 D}^{1, *}$ \\ Yana Ivashkevich $\mathbb{D}^{1, *}$ \\ Yaroslav Chernov ${ }^{2}$ \\ Denis Chinenov ${ }^{2}$ \\ Evgeniy Shpot ${ }^{2}$ \\ Alexander A Bessonov $\mathbb{I D}^{3}$ \\ Bouthaina S Dabaja ${ }^{4}$ \\ Sergej Konoplev' \\ 'Department of Hematopathology, The \\ University of Texas MD Anderson Cancer \\ Center, Houston, TX, 77030, USA; \\ ${ }^{2}$ Institute for Urology and Reproductive \\ Health, I.M. Sechenov First Moscow State \\ Medical University, Moscow, Russia; \\ ${ }^{3}$ Breast Cancer Department, NMRC N. \\ N. Petrov Research Institute of Oncology \\ of the Ministry of Healthcare, \\ St. Petersburg, Russia; ${ }^{4}$ Radiation \\ Oncology Department, The University of \\ Texas MD Anderson Cancer Center, \\ Houston, TX, USA
}

*These authors contributed equally to this work

Correspondence: Sergej Konoplev Department of Hematopathology, The University of Texas MD Anderson Cancer Center, Houston, TX, 77030, USA

Tel + I 7|3-792-4III

Fax + 1 713-794-1800

Email SKonople@mdanderson.org

\begin{abstract}
Extranodal marginal zone lymphoma of mucosa-associated lymphoid tissue (MALT lymphoma) of the urinary bladder is an extremely rare entity accounting for $0.2 \%$ of all malignant urinary bladder neoplasms, and the diagnosis could be challenging. We present here a patient with urinary bladder MALT lymphoma and review of all published case reports in the literature. We summarized the reported immunophenotype of the neoplasm, ancillary studies, therapy, and follow-up for all 59 patients in the table. The median patients' age was 57 years-old (range, 17 to 88), with female predominance in 50 of 59 patients representing a 1:5.6 ratio. Geographical distribution of the reported patients was as follows: 22 from Asia, of which more than a half (16) originated from Japan; 28 from Europe, of which 19 reported from the United Kingdom, and 3 patients were reported from the United States (including our patient). Twenty-three (77\%) of 30 patients, for whom their clinical presentation was recorded, had symptoms of cystitis; Escherichia coli was the most common pathogen. We concluded that a prominent female predominance, uneven geographic distribution of urinary bladder MALT lymphoma, and a success of antibacterial therapy in selected cases suggest the link between urinary tract infection and urinary bladder MALT lymphoma.
\end{abstract}

Keywords: MALT lymphoma, urinary bladder, cystitis, urinary tract infection

\section{Introduction}

Extranodal marginal zone lymphoma of mucosa-associated lymphoid tissue (MALT lymphoma) is a low-grade B cell lymphoma which accounts for 7-8\% of all B cell lymphomas. ${ }^{1}$ The stomach is the most common organ involved by MALT lymphoma (about 35\%), followed by other anatomical locations: eyes and ocular adnexa, skin, lungs, salivary glands, breasts, and thyroid. ${ }^{2}$

The first definitive report of MALT lymphoma in urinary bladder was published in 1990 by Kuhara and colleagues. ${ }^{3}$ MALT lymphoma of the urinary bladder is an extremely rare entity and accounts to less than $1 \%$ of all nonHodgkin lymphomas and $0.2 \%$ of all malignant urinary bladder neoplasms. ${ }^{4}$ The majority of patients present with hematuria and/or dysuria. The differential diagnosis could be very broad and includes inflammatory lesions, bladder carcinoma, and infections. ${ }^{5}$ 
Some authors hypothesized that chronic antigenic stimulation and lymphoid hyperplasia caused by Escherichia coli or other bacterial infections might be a precursor of bladder MALT lymphomas. ${ }^{6}$ The positive experience in antibiotics treatment of MALT lymphoma of bladder might serve as an indirect support of this hypothesis. ${ }^{7-11}$

Herein, we report and discuss a case of primary bladder MALT lymphoma and summarize other cases reported in the literature.

\section{Case Presentation}

A 58-year-old Caucasian female with history of hypertension, asthma, rheumatoid arthritis, irritable bowel syndrome, colon polyps, depression, and menopausal syndrome presented to the clinic with nonspecific urinary symptoms: dysuria, nocturia, and urinary frequency. There were no $\mathrm{B}$ symptoms identified. The $\mathrm{CT}$ demonstrated multiple thickening on the anterior and posterior bladder walls (Figure 1A and B). Consequently, patient underwent a cystoscopy evaluation with transurethral resection of bladder tumor (TURBT), which revealed $2.5 \times 2.5 \mathrm{~cm}$ mass on posterior bladder wall distally from the trigone. The mass did not have characteristic bladder cancer architecture. The patient signed the informed consent/authorization for participation in research which includes the permission to collect and use the information from medical records, imaging studies, medical photographs, pathology images, and study results for future research projects and publications. A copy of the signed consent is kept on file in the patient electronic records.

\section{Pathological Findings}

The histologic sections showed a dense lymphocytic proliferation beneath the urothelial surface of urinary bladder mucosa. Within the lymphoid proliferation, a monomorphic population of small lymphocytes having moderately abundant, pale staining cytoplasm was dominant. Regularly scattered, reactive lymphoid follicles were prominent within the mucosal lymphoid proliferation. The neoplastic lymphocytic proliferation had a perifollicular infiltration pattern.

Multiples immunohistochemical studies on biopsy material were performed and showed that neoplastic cells were positive for CD20, CD79a, BCL-2, and immunoglobulin kappa light chain (weak) confirming the clonality of the neoplastic cells; the neoplastic cells were negative for CD5, CD10, and immunoglobulin lambda light chain (Figure 2).

The diagnosis of MALT lymphoma of urinary bladder floor was established. No additional molecular studies were performed.

\section{Treatment/Follow-Up}

The patient received 4 cycles of Rituximab and achieved complete remission seen on the pelvic computed tomography (CT) (Figure 3). The patient showed no signs of disease with the last follow-up more than 10 years after original diagnosis.

\section{Literature Review}

The literature review was initiated starting with 1990 when Kuhara and colleagues reported what was eventually called "the first definitive report of a MALT lymphoma of the urinary bladder" ${ }^{3,12}$ After a systemic search of the PubMed database for primary MALT lymphoma involving urinary bladder, the search identified 42 reports with a total of 58 patients. ${ }^{3,5-45}$ The available information about diagnostic immunohistochemistry, clinical manifestations, treatments, and outcomes of these cases along with a current case is summarized in Table 1.,5-45 There was a strong female predominance with 50 of 59 patients being females with a male:female ratio of 1:5.6. The median patients' age was 57 years-old (range, 17 to 88). A significant proportion of cases (22 total cases) was
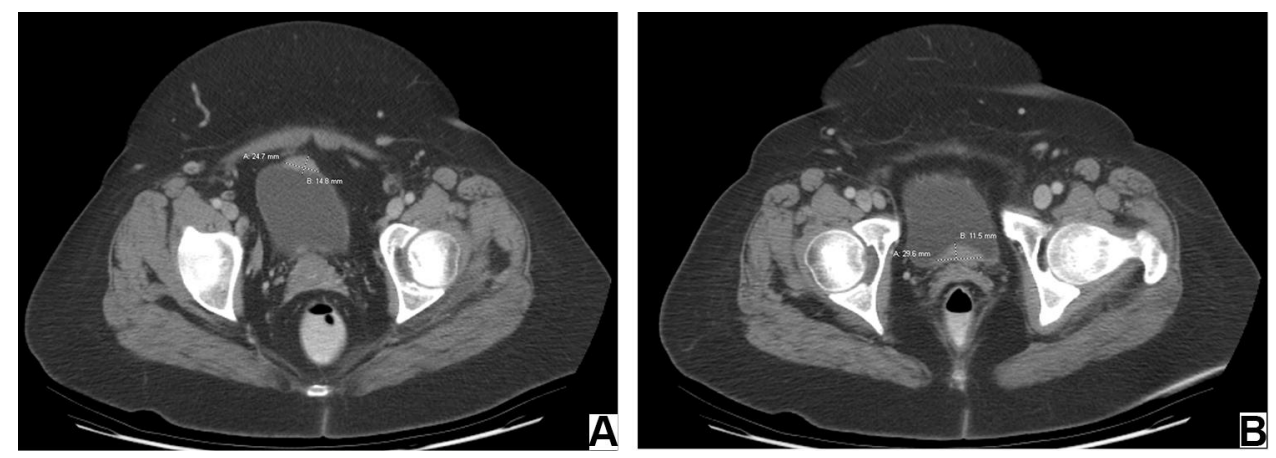

Figure I Axial CT images showing multiple abnormal nodular thickening on the anterior $(\mathbf{A})$ and posterior $(\mathbf{B})$ bladder walls. 

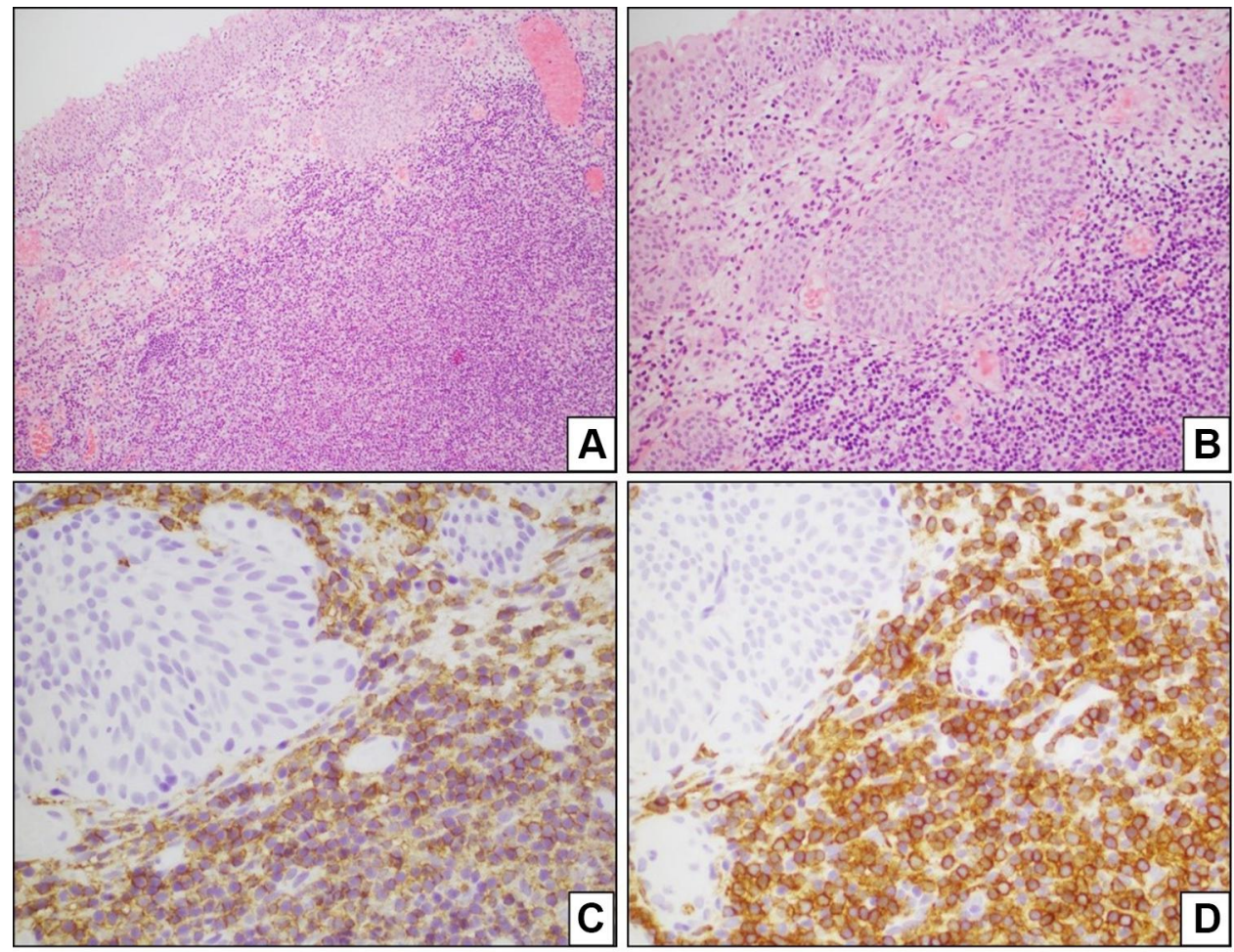

Figure 2 Bladder MALT lymphoma. The histologic sections show a dense, abnormal lymphocytic proliferation right beneath the urothelial surface of urinary bladder mucosa $(\mathbf{A}$ and $\mathbf{B})$ the neoplastic cells are positive for CD20 (C) and CD79a (D).

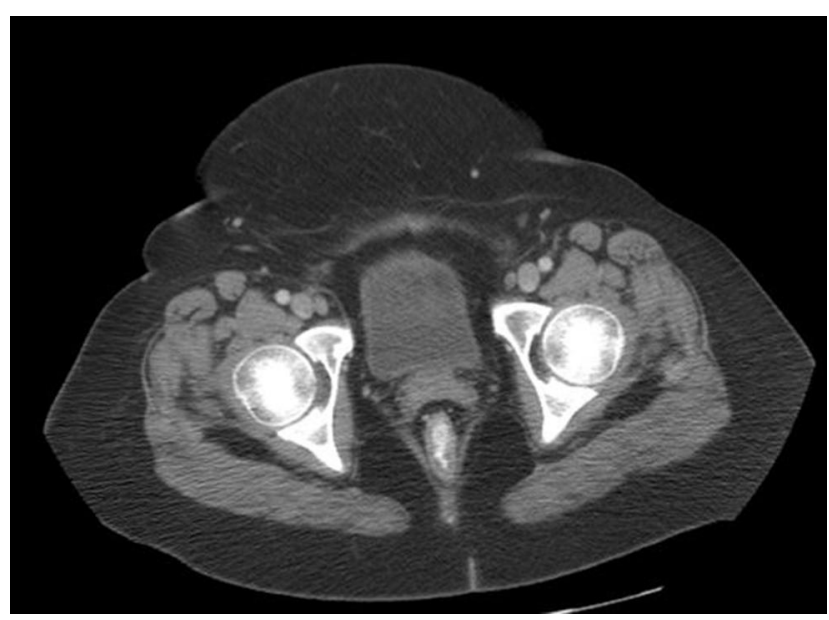

Figure 3 Axial CT image of depicting the bladder and showing no evidence of abnormal nodularity 3 months later.

reported from in Asia, of which more than half (16) originated from Japan 3 ,9,10,16,18,22-24,28,31,33,36,41,42,44,45 According to some of the available reports there more publications in Japanese literature which we could not find in PubMed. ${ }^{23,41}$ The cases reported from Asia showed even a higher female predominance where only one of 22 patients was a male. ${ }^{18}$ United Kingdom was another location with significant number of patients and accounted for
19 out of 28 European cases. ${ }^{8,12,17,20,21,25,40}$ Including our patient, only 3 patients were reported in the USA. ${ }^{32,34}$ All patients had some urinary symptoms at presentation which led to the diagnosis. Hematuria was the most common presenting symptom and was previously reported from $50.9 \%$ to $75 \%$. Twenty three of 30 patients, for whom the information regarding presence or absence of cystitis was available, had cystitis; Escherichia coli was the most common pathogen. ${ }^{21,38} \mathrm{Xu}$ and colleagues found that most patients, 76.5\%, had a bladder solid mass on presentation. ${ }^{38}$ None of patients demonstrated "B" group symptoms such as weight loss, fever, or night sweats.

Only one case of MALT lymphoma with CD5 expression was reported. ${ }^{10}$ The clonality for heavy-chain $\operatorname{IgH}$ gene rearrangement by PCR was studied in 10 cases and was found to be clonally rearranged. ${ }^{6,8,16,24,28,29,39}$

Cytogenetic studies were reported in two cases. ${ }^{11,29}$ Krober and colleagues found that the first case was negative for $\mathrm{t}(14: 18)$ and positive for trisomy 3 , while second case showed translocation $\mathrm{t}(11 ; 18)(\mathrm{q} 21 ; \mathrm{q} 21)$, trisomy 3 , and trisomy $18 .^{29}$

Treatment approaches were variable including surgical excision, antibiotics, chemotherapy, radiation, or combined modality. Six patients were successfully treated 


\begin{tabular}{|c|c|c|c|c|c|c|c|}
\hline 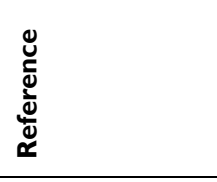 & 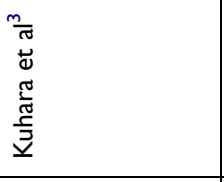 & 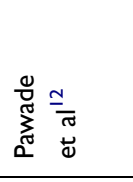 & 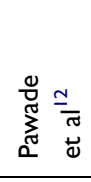 & 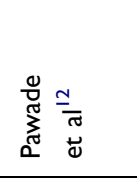 & 离 $\frac{}{\pi}$ & 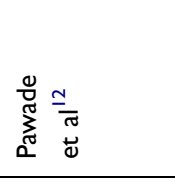 & 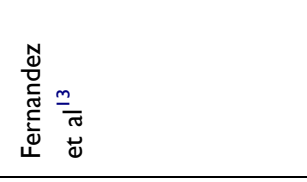 \\
\hline 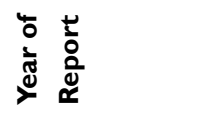 & $\frac{8}{2}$ & $\stackrel{m}{\sigma}$ & $\stackrel{m}{\sigma}$ & $\stackrel{m}{\sigma}$ & $\stackrel{m}{\sigma}$ & $\stackrel{m}{\sigma}$ & よ̊ \\
\hline 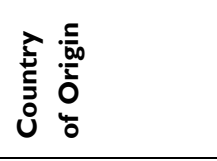 & 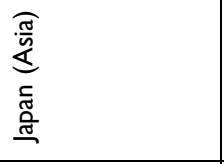 & 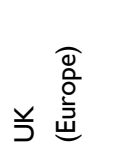 & 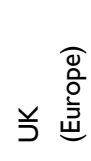 & 弚 & 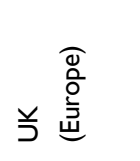 & 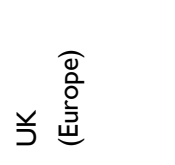 & 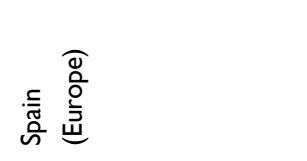 \\
\hline 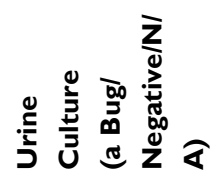 & 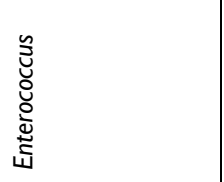 & $\lesssim$ & $\overleftarrow{z}$ & 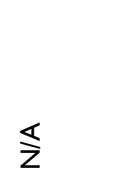 & $\overleftarrow{z}$ & 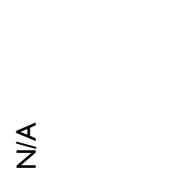 & 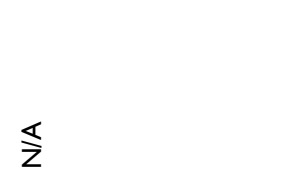 \\
\hline 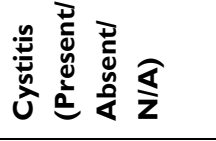 & 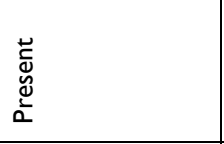 & 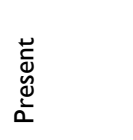 & 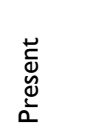 & $\$$ & $\$$ & 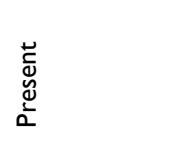 & 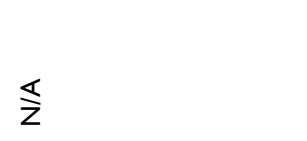 \\
\hline 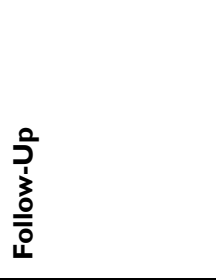 & 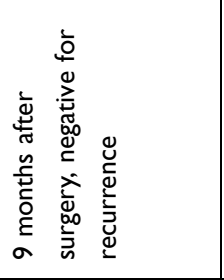 & 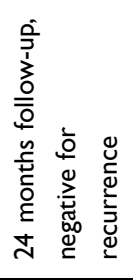 & 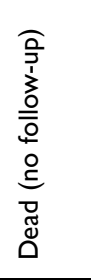 & 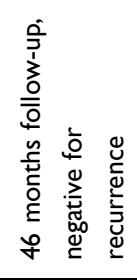 & 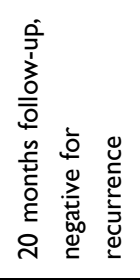 & 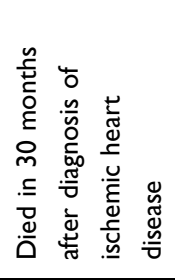 & 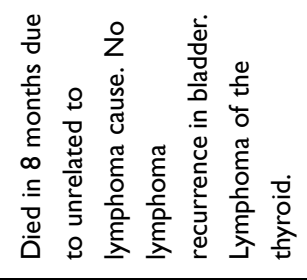 \\
\hline 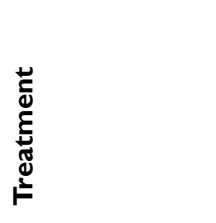 & 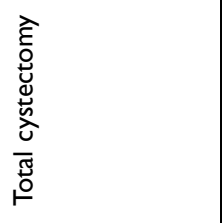 & 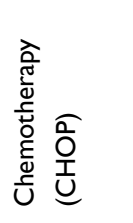 & 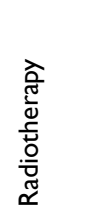 & 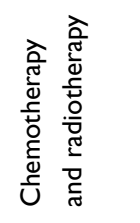 & 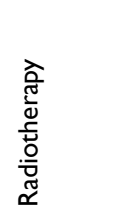 & 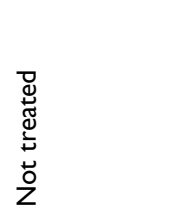 & 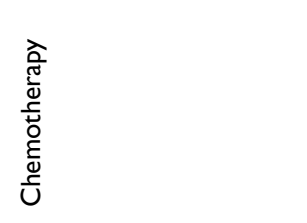 \\
\hline 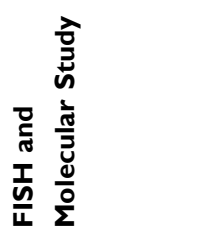 & $\$$ & $\$$ & $\lesssim$ & 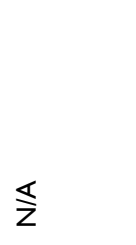 & 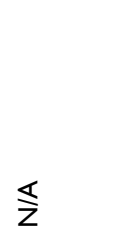 & 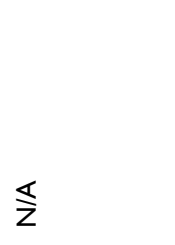 & 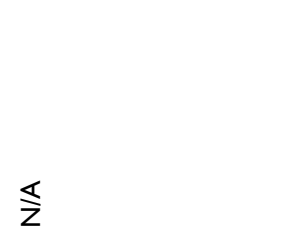 \\
\hline 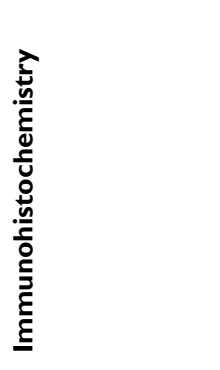 & 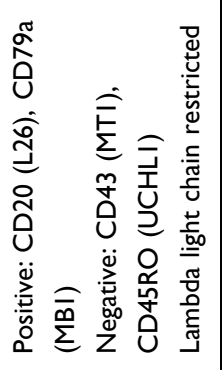 & 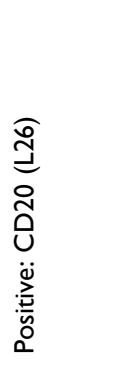 & 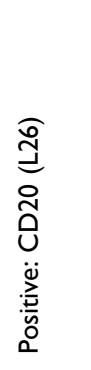 & 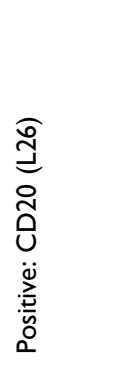 & 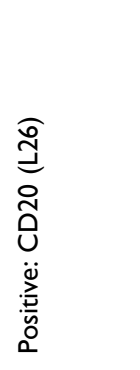 & 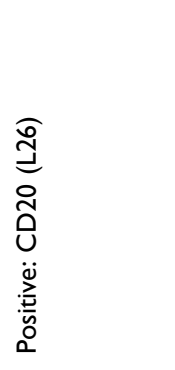 & 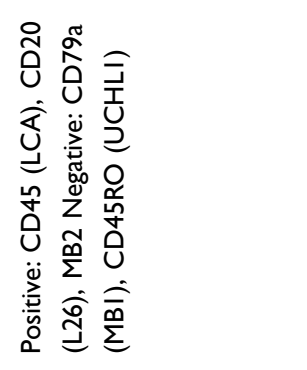 \\
\hline ڤั & 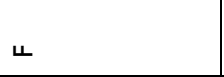 & ч & ч & 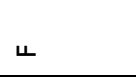 & ч & $\Sigma$ & ч \\
\hline 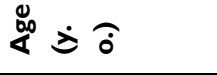 & 亡̊ & $\hat{0}$ & $\stackrel{N}{\star}$ & ה & 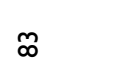 & $\infty$ & $\stackrel{M}{\wedge}$ \\
\hline 仓̊ & - & $N$ & $m$ & 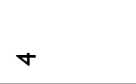 & in & 0 & $r$ \\
\hline
\end{tabular}




\begin{tabular}{|c|c|c|c|c|c|c|c|c|c|}
\hline 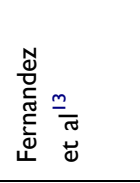 & 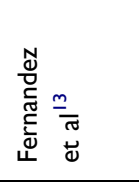 & 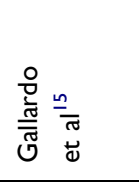 & 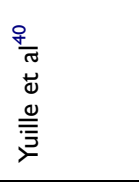 & 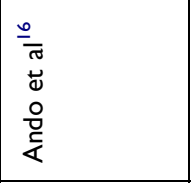 & 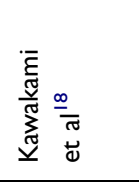 & 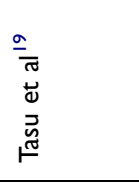 & 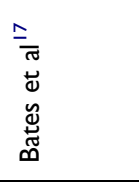 & 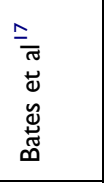 & 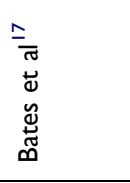 \\
\hline$\stackrel{\circ}{\varrho}$ & $\stackrel{\circ}{\circ}$ & $\stackrel{\text { 品 }}{\stackrel{-}{2}}$ & $\stackrel{\infty}{\circ}$ & $\stackrel{\sigma}{\sigma}$ & : & : & : & ঃ্ণ & : \\
\hline 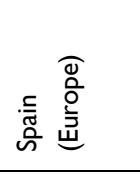 & 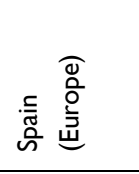 & 严 & כ & 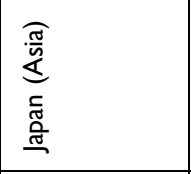 & 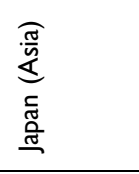 & 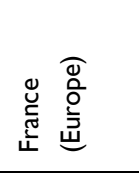 & 兰总 & 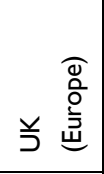 & 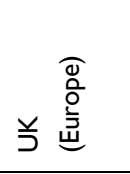 \\
\hline$\widehat{\Sigma}$ & $\widehat{\Sigma}$ & $\stackrel{\mathbb{z}}{\mathrm{z}}$ & 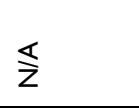 & 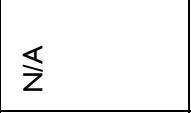 & $\widehat{\Sigma}$ & $\frac{\mathbb{s}}{z}$ & $\widehat{\Sigma}$ & $\widehat{\Sigma}$ & $\stackrel{\mathbb{s}}{\mathbf{z}}$ \\
\hline 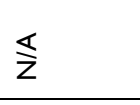 & 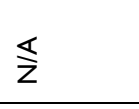 & $\underline{\Sigma}$ & $\$$ & 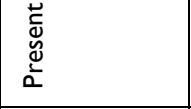 & $\widehat{\Sigma}$ & $\frac{\pi}{z}$ & $\frac{\pi}{z}$ & $\widehat{\Sigma}$ & $\widehat{\Sigma}$ \\
\hline 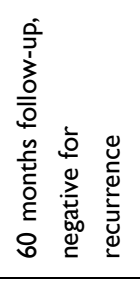 & 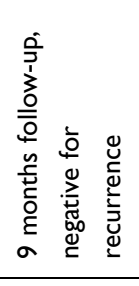 & 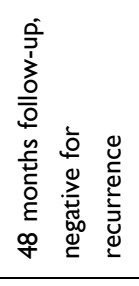 & 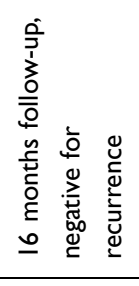 & 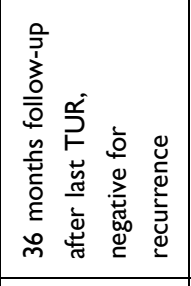 & 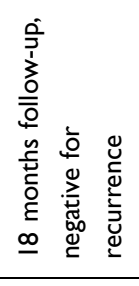 & 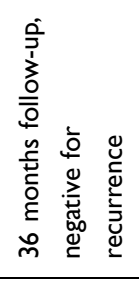 & 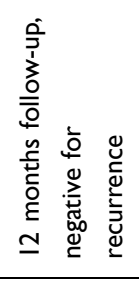 & $\begin{array}{l}0 \\
\frac{0}{1} \\
\frac{3}{0} \\
\frac{0}{0} \\
0 \\
z \\
\end{array}$ & 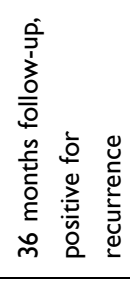 \\
\hline 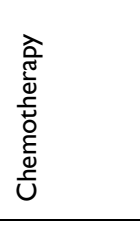 & 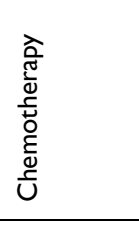 & 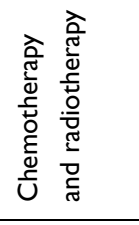 & 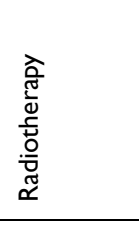 & 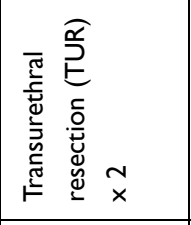 & 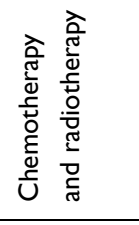 & 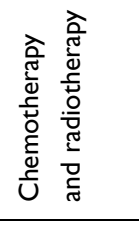 & 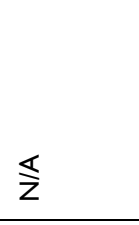 & 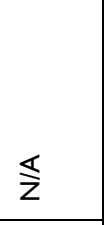 & $\widehat{\Sigma}$ \\
\hline 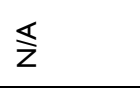 & 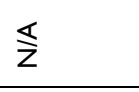 & 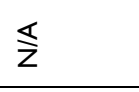 & 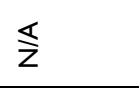 & 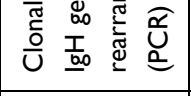 & 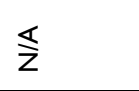 & 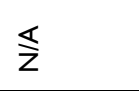 & $\stackrel{\mathbb{z}}{\mathrm{z}}$ & $\widehat{\Sigma}$ & $\widehat{\Sigma}$ \\
\hline 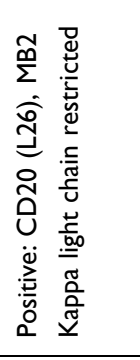 & 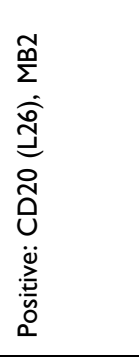 & $\widehat{\Sigma}$ & 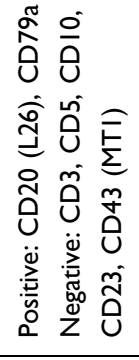 & 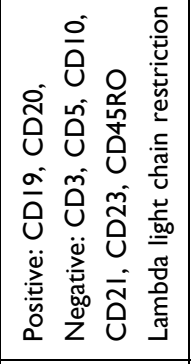 & $\widehat{\Sigma}$ & $\underline{\Sigma}$ & 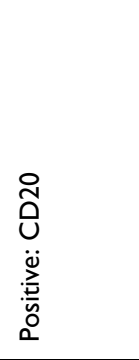 & 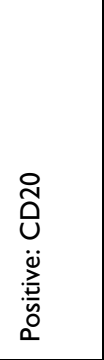 & 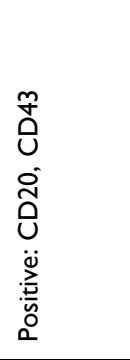 \\
\hline 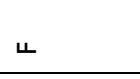 & 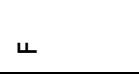 & 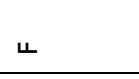 & 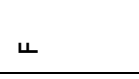 & 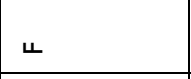 & $\Sigma$ & 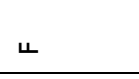 & 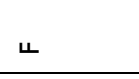 & 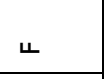 & 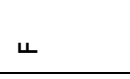 \\
\hline in & $\mathfrak{n}$ & $R$ & $\infty$ & R & $\hat{\lambda}$ & $\mathfrak{n}$ & $\stackrel{8}{\circ}$ & 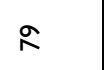 & in \\
\hline$\infty$ & $\sigma$ & $\stackrel{\circ}{ }$ & $=$ & $\simeq$ & $\underline{m}$ & \pm & $\underline{\underline{ }}$ & 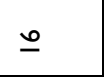 & $\simeq$ \\
\hline
\end{tabular}




\begin{tabular}{|c|c|c|c|c|c|c|c|}
\hline 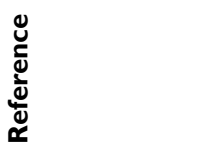 & 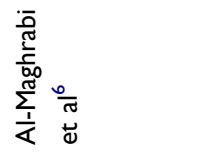 & 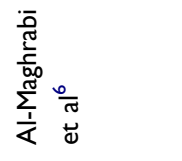 & 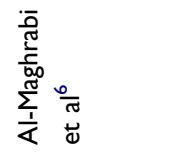 & 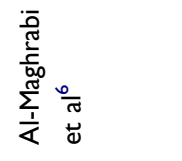 & 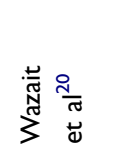 & 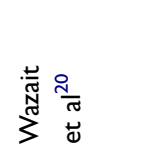 & 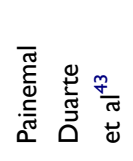 \\
\hline 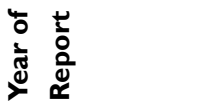 & ষ্ণ & চ্ণ & চ্ণ & চ্ণ & ర্ণ & ర্ণ & ষ্ণ \\
\hline 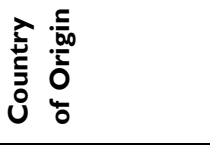 & 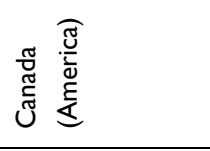 & 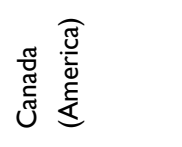 & 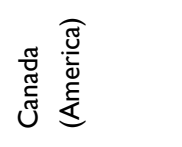 & 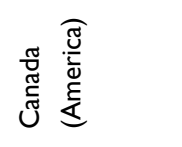 & 弚 & 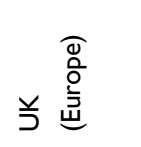 & 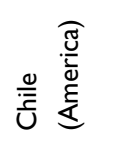 \\
\hline 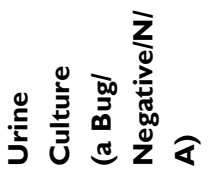 & 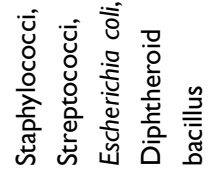 & 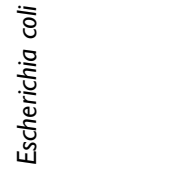 & 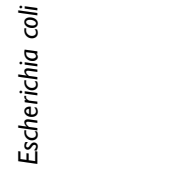 & 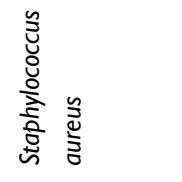 & 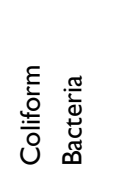 & 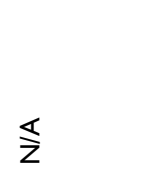 & $\overleftarrow{z}$ \\
\hline 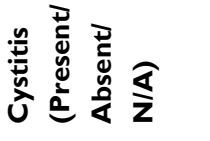 & 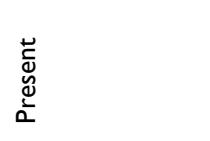 & 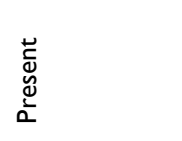 & 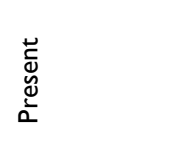 & 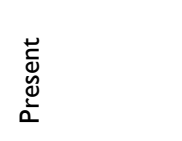 & 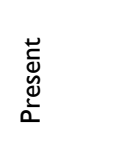 & $\$$ & 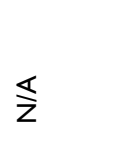 \\
\hline 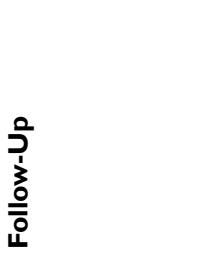 & 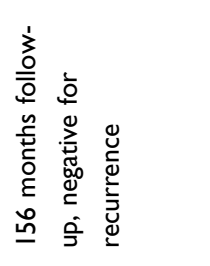 & 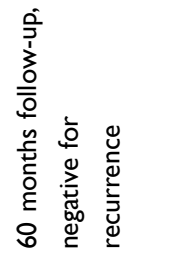 & 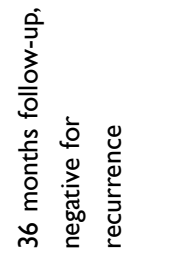 & 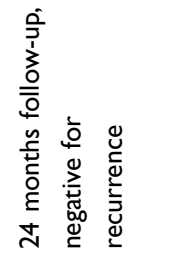 & 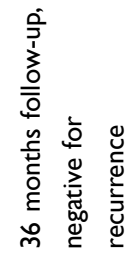 & 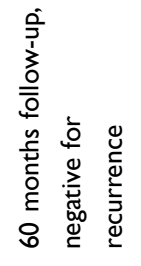 & 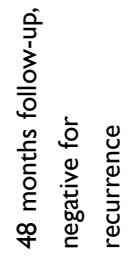 \\
\hline 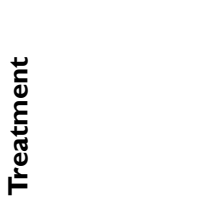 & 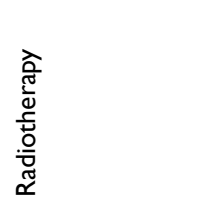 & 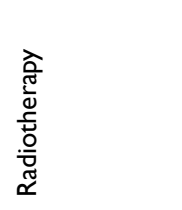 & 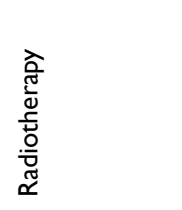 & 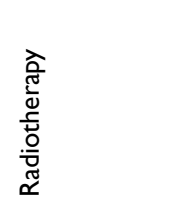 & 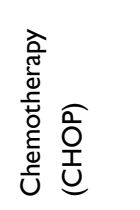 & 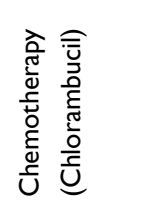 & 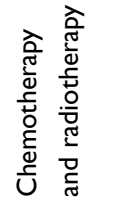 \\
\hline 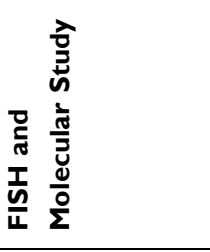 & 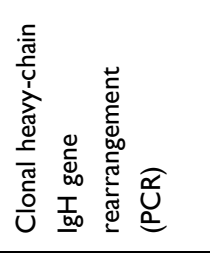 & 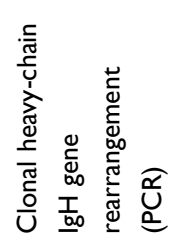 & 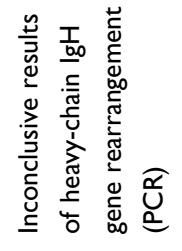 & 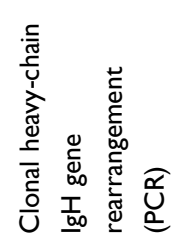 & 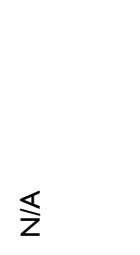 & $\overleftarrow{Z}$ & 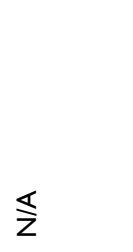 \\
\hline 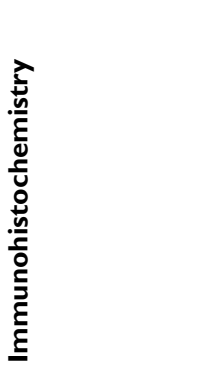 & 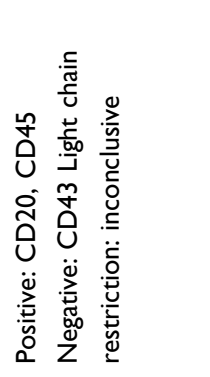 & 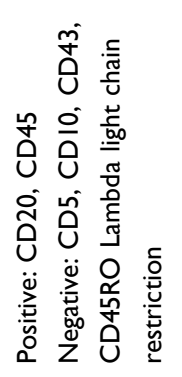 & 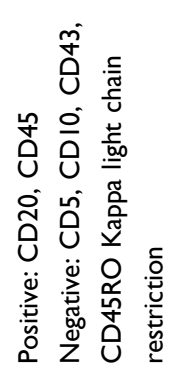 & 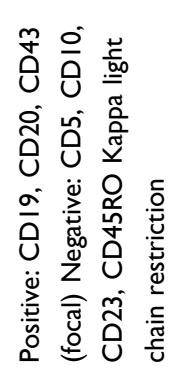 & 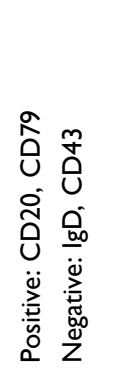 & 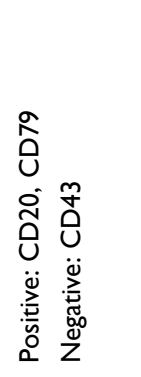 & 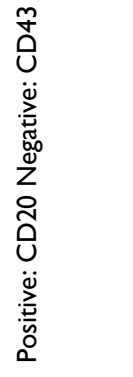 \\
\hline ڤึ & 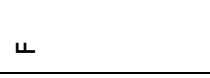 & \llcorner & 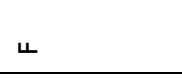 & $\Sigma$ & ᄂ & ᄂ & \llcorner \\
\hline 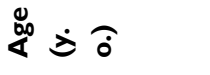 & ț & oิ & $\mathbb{N}$ & $\widetilde{\sigma}$ & $\stackrel{\sim}{0}$ & $R$ & $R$ \\
\hline ü & $\underline{\infty}$ & $\underline{\sigma}$ & సి & $\bar{N}$ & $\pi$ & $\stackrel{\sim}{\sim}$ & $\stackrel{ \pm}{\sim}$ \\
\hline
\end{tabular}




\begin{tabular}{|c|c|c|c|c|c|c|c|c|}
\hline 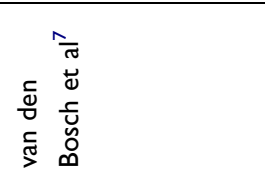 & $\begin{array}{l}\overline{\grave{\nu}} \\
\stackrel{\overline{0}}{\overline{0}} \\
\frac{\bar{y}}{\tilde{U}}\end{array}$ & 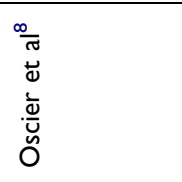 & 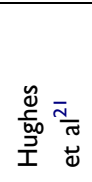 & 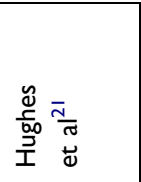 & 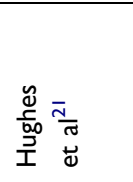 & 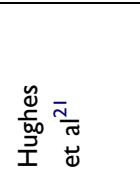 & 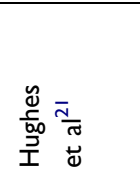 & 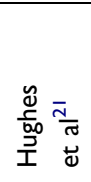 \\
\hline ర్రి & ర్రి & ర్రి & ڤ్రి & ڤ్రి & ڤ్రి & 옹 & ڤ్రి & ڤ్రి \\
\hline 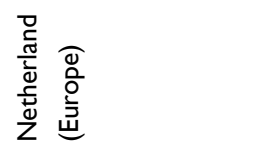 & 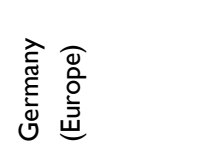 & 弚 & 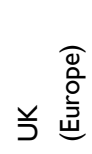 & 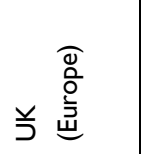 & 弚总 & 弚 & 弚 & 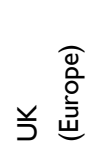 \\
\hline 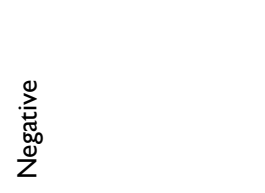 & $\overleftarrow{z}$ & 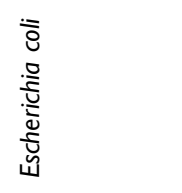 & $\lesssim$ & $\overleftarrow{z}$ & $\stackrel{\nwarrow}{z}$ & $\overleftarrow{z}$ & 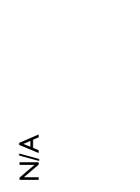 & $\overleftrightarrow{z}$ \\
\hline $\begin{array}{l}\text { पू⿱艹 } \\
\text { 晏 }\end{array}$ & 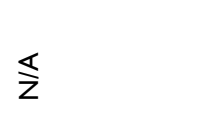 & 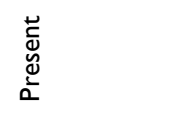 & 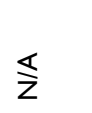 & 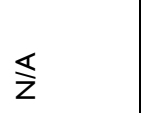 & 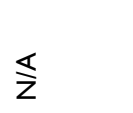 & 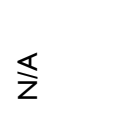 & 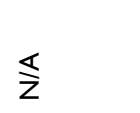 & 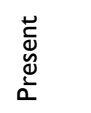 \\
\hline 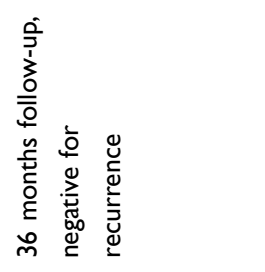 & 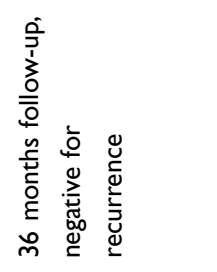 & 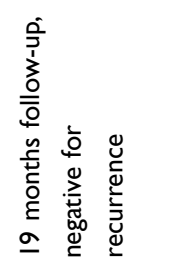 & 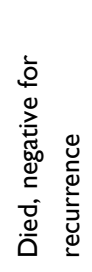 & 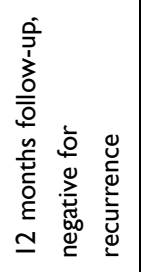 & 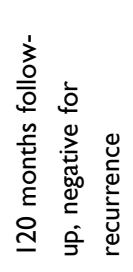 & 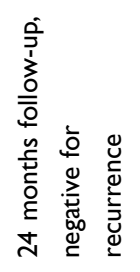 & 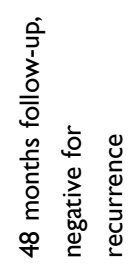 & 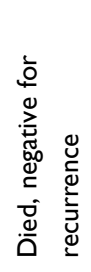 \\
\hline 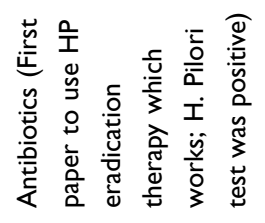 & 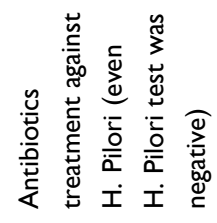 & 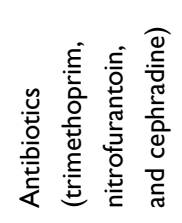 & 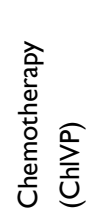 & 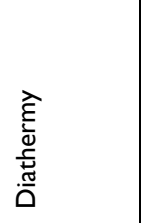 & 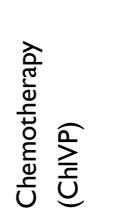 & 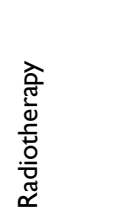 & 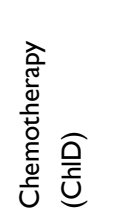 & 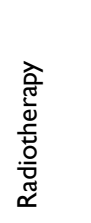 \\
\hline $\mathbb{z}$ & 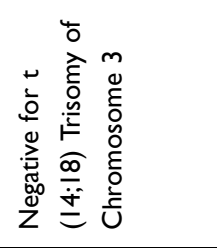 & 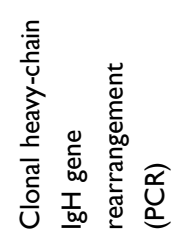 & 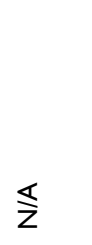 & $\stackrel{\nwarrow}{z}$ & $\$$ & $\stackrel{\nwarrow}{z}$ & 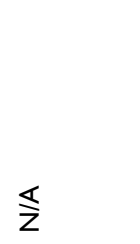 & $\lesssim$ \\
\hline 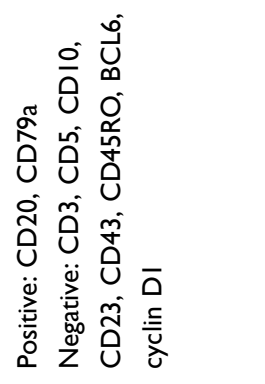 & 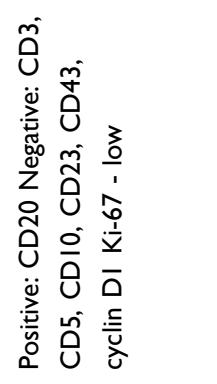 & 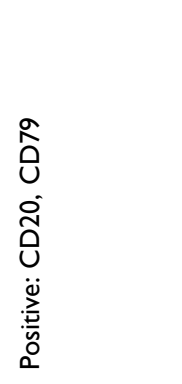 & 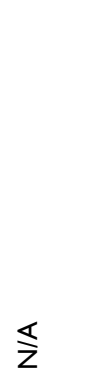 & $\$$ & 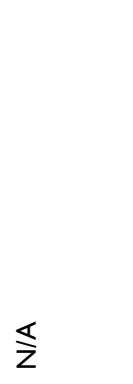 & $\stackrel{\nwarrow}{z}$ & $\overleftrightarrow{\Sigma}$ & 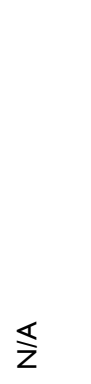 \\
\hline$\Sigma$ & $\Sigma$ & ч & ч & 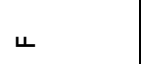 & $\Sigma$ & ч & $\Sigma$ & ч \\
\hline in & in & $\stackrel{\infty}{\wedge}$ & ఎ & $\bar{\infty}$ & $\stackrel{\infty}{\sim}$ & $\stackrel{2}{\circ}$ & $\therefore$ & $\stackrel{\circ}{\circ}$ \\
\hline$\stackrel{\sim}{\sim}$ & $\stackrel{2}{\sim}$ & $\hat{\lambda}$ & $\stackrel{\infty}{\sim}$ & సి & 이 & $\bar{m}$ & $\widetilde{m}$ & $\stackrel{m}{m}$ \\
\hline
\end{tabular}




\begin{tabular}{|c|c|c|c|c|c|c|c|c|}
\hline 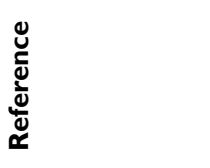 & 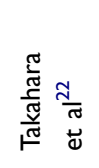 & 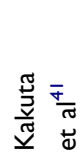 & 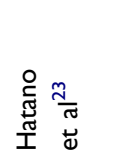 & 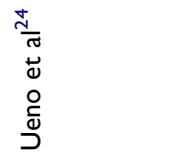 & 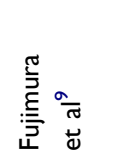 & 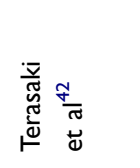 & 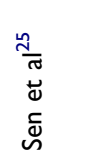 & 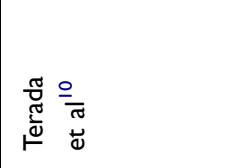 \\
\hline 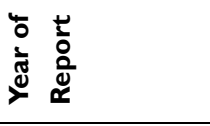 & ڤ్ & ठั่ & ڤ્̀ & ڤે̀ & ळ্ं & ळ̊ํํ & $\frac{\text { 음 }}{2}$ & $\overline{\bar{i}}$ \\
\hline 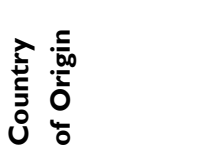 & 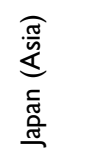 & 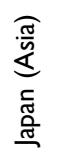 & 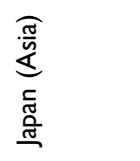 & 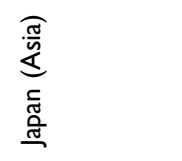 & 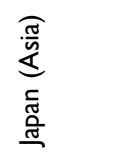 & 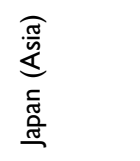 & 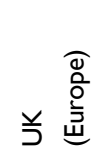 & 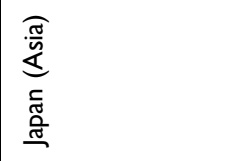 \\
\hline 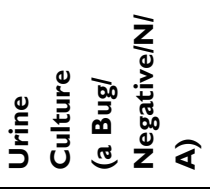 & 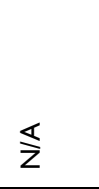 & $\$$ & 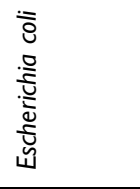 & 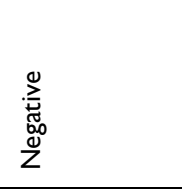 & 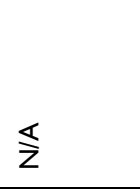 & 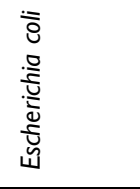 & 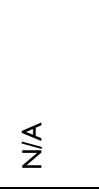 & 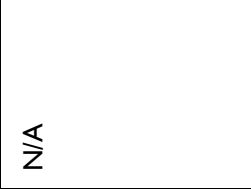 \\
\hline 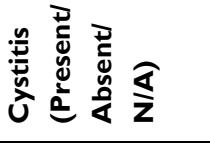 & 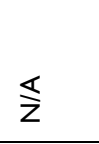 & $\$$ & 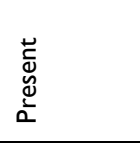 & 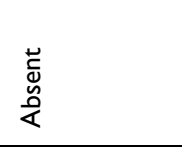 & 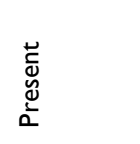 & 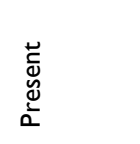 & $\$$ & $\widehat{\Sigma}$ \\
\hline 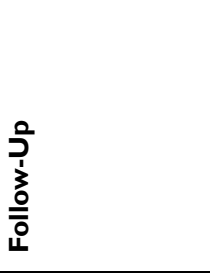 & 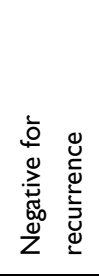 & 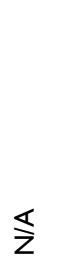 & 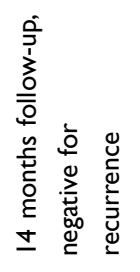 & 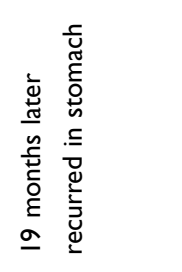 & 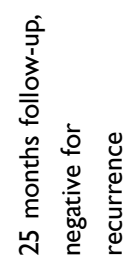 & 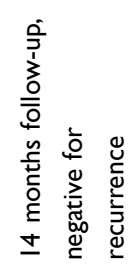 & 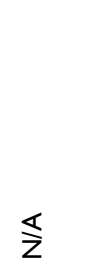 & 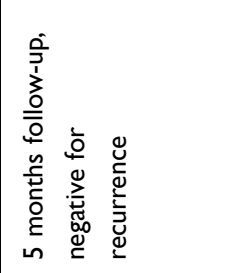 \\
\hline 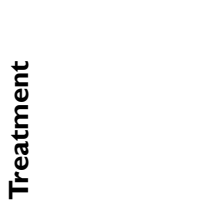 & 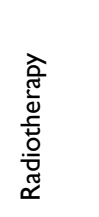 & 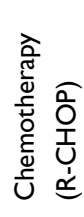 & 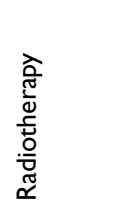 & 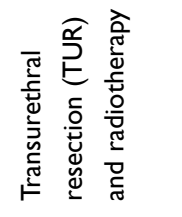 & 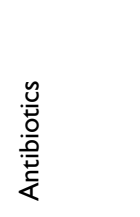 & 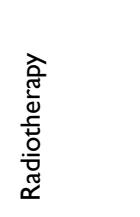 & 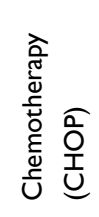 & 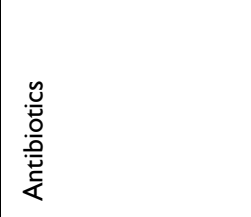 \\
\hline 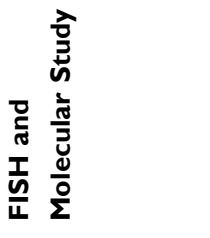 & 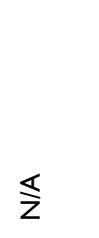 & 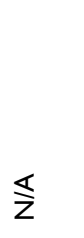 & $\$$ & 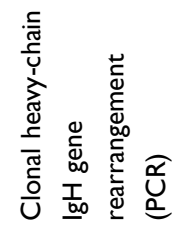 & 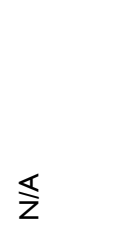 & 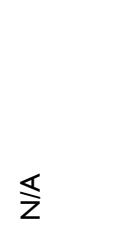 & 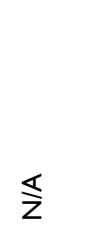 & 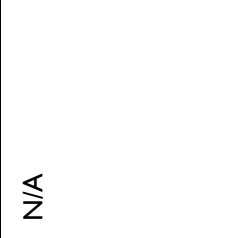 \\
\hline 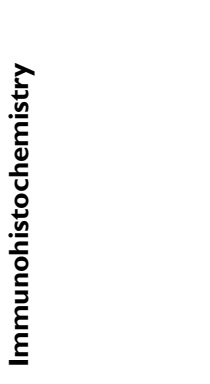 & 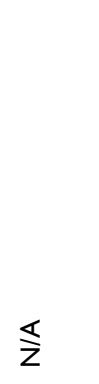 & $\$$ & $\$$ & 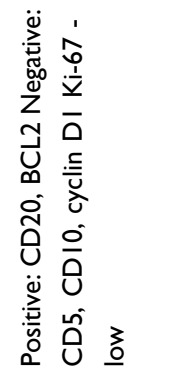 & 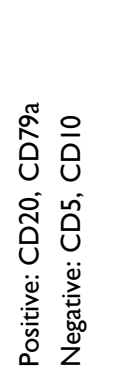 & 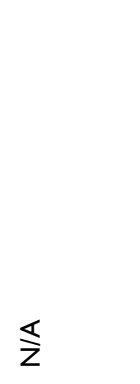 & 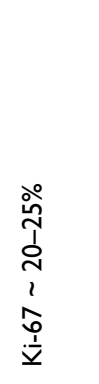 & 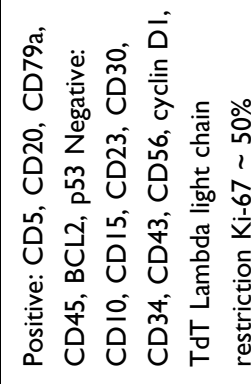 \\
\hline ڤ్ & น & 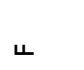 & ᄂ & ч & ч & น & ए & ч \\
\hline 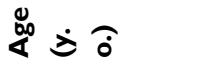 & $\stackrel{\llcorner}{\infty}$ & $\stackrel{\infty}{\infty}$ & 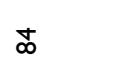 & t & o & ఫ & $\bar{m}$ & $\left.\right|_{\infty} ^{\infty}$ \\
\hline نू & 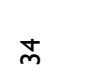 & $\stackrel{\sim}{m}$ & లి & $\hat{m}$ & $\stackrel{\infty}{m}$ & 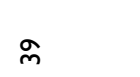 & q & $\bar{\sigma}$ \\
\hline
\end{tabular}




\begin{tabular}{|c|c|c|c|c|c|c|}
\hline 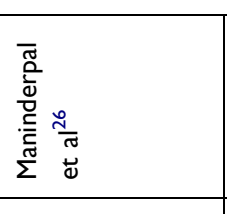 & 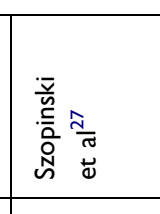 & 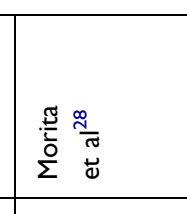 & 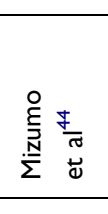 & 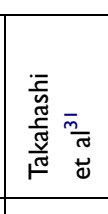 & 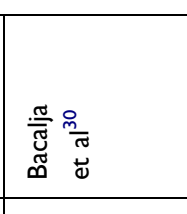 & 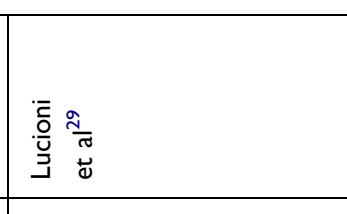 \\
\hline$\overline{\bar{\alpha}}$ & $\overline{\bar{\alpha}}$ & $\bar{a}$ & $\frac{\mathrm{m}}{\mathrm{a}}$ & $\frac{m}{a}$ & $\frac{m}{a j}$ & $\frac{m}{a}$ \\
\hline 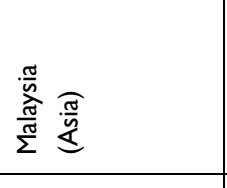 & 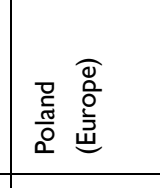 & 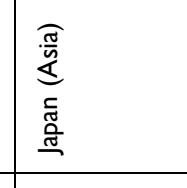 & 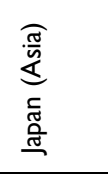 & 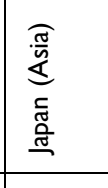 & 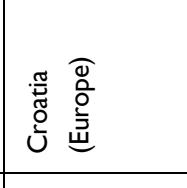 & 产高 \\
\hline$\frac{\pi}{z}$ & $\frac{\pi}{z}$ & $\frac{\underline{x}}{\bar{z}}$ & $\frac{\pi}{z}$ & $\frac{\pi}{z}$ & 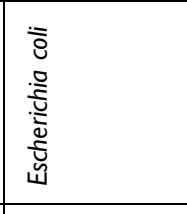 & 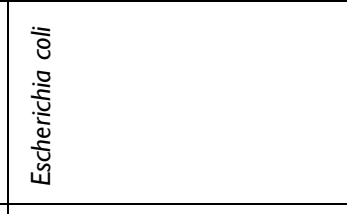 \\
\hline 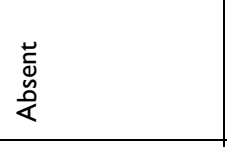 & $\frac{\pi}{2}$ & \begin{tabular}{|l|} 
\\
奒 \\
\end{tabular} & 密 & 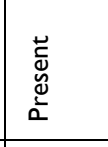 & 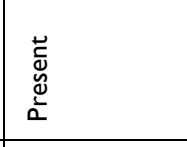 & 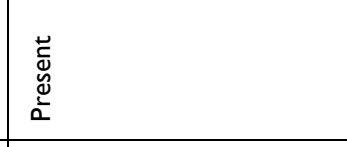 \\
\hline 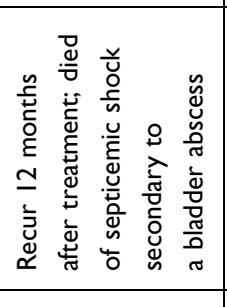 & 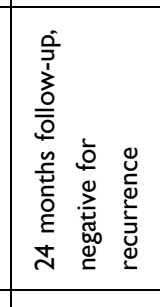 & 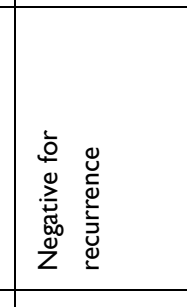 & $\frac{\pi}{z}$ & $\frac{\pi}{2}$ & $\frac{\pi}{z}$ & 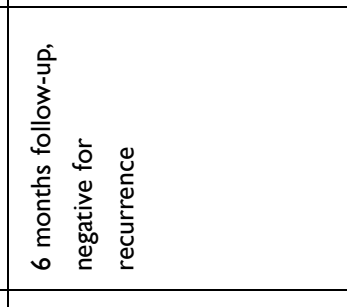 \\
\hline 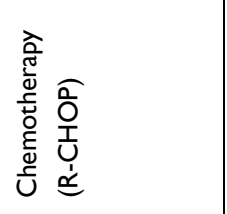 & 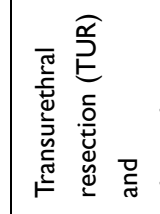 & & 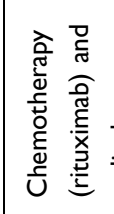 & 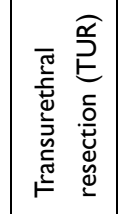 & 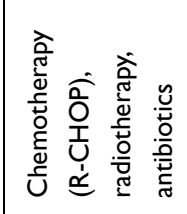 & 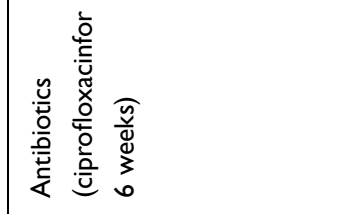 \\
\hline$\frac{\underline{x}}{\mathbf{z}}$ & $\frac{\pi}{z}$ & 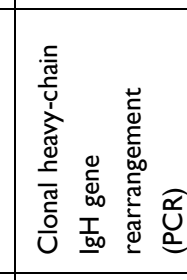 & $\frac{\pi}{2}$ & $\frac{\pi}{z}$ & $\frac{\pi}{2}$ & 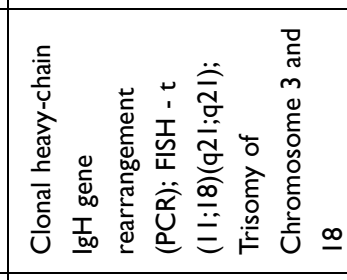 \\
\hline 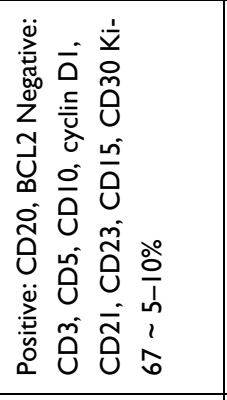 & 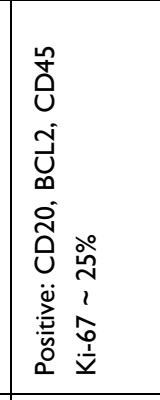 & 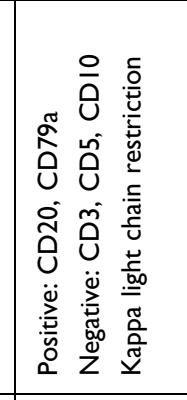 & $\frac{\pi}{z}$ & 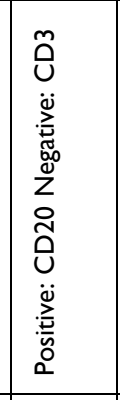 & 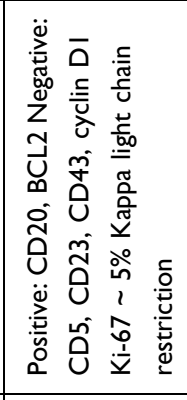 & 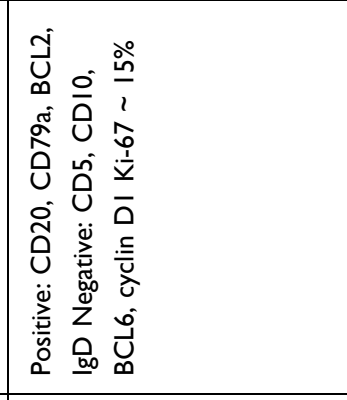 \\
\hline$\Perp$ & 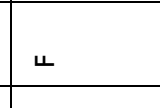 & 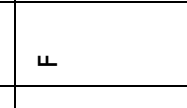 & 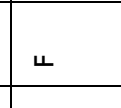 & 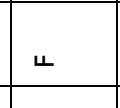 & $\Sigma$ & 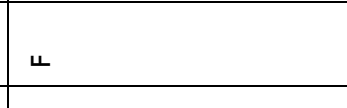 \\
\hline$\therefore$ & $=$ & 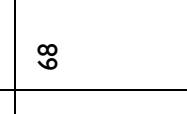 & $\approx$ & $=$ & 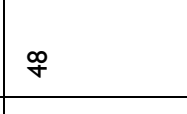 & $\approx$ \\
\hline 7 & F & $\ddagger$ & 7 & 8 & F & क् \\
\hline
\end{tabular}




\begin{tabular}{|c|c|c|c|c|c|c|c|}
\hline 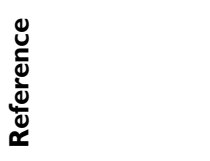 & 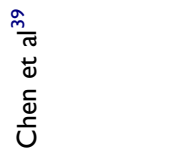 & 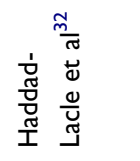 & 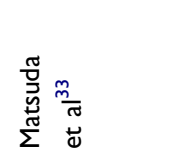 & 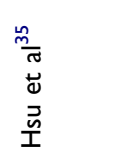 & 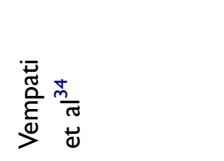 & 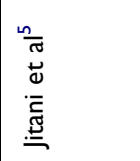 & 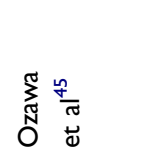 \\
\hline 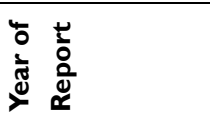 & $\frac{\nabla}{i}$ & $\frac{\pi}{\grave{N}}$ & $\frac{d}{0}$ & $\stackrel{n}{\circ}$ & $\frac{n}{\grave{N}}$ & 윰 & $\frac{\infty}{i}$ \\
\hline 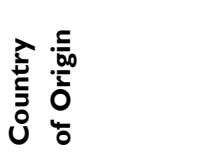 & 谞 & 芯 & 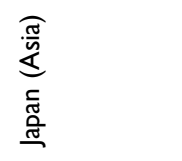 & 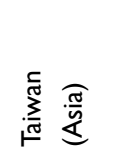 & 芩 & 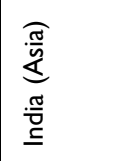 & 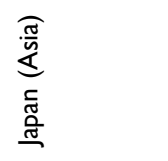 \\
\hline 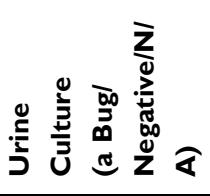 & 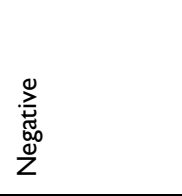 & 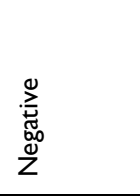 & 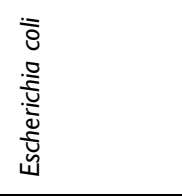 & 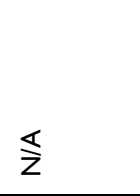 & $\$$ & 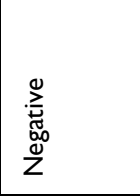 & 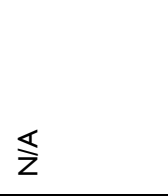 \\
\hline 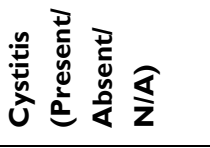 & 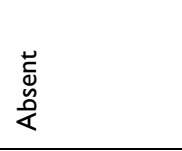 & 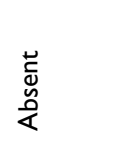 & 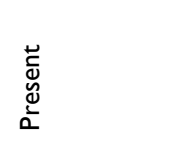 & 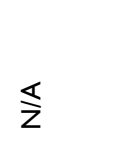 & 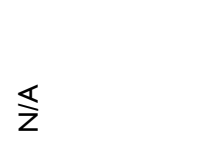 & 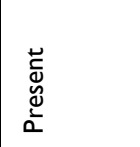 & $\stackrel{\varangle}{z}$ \\
\hline $\begin{array}{l}\sum_{0}^{0} \\
\vdots \\
0 \\
0 \\
\end{array}$ & 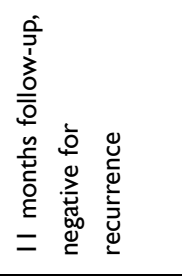 & 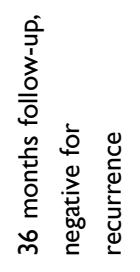 & 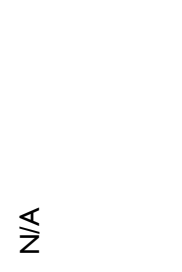 & 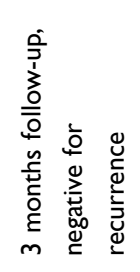 & 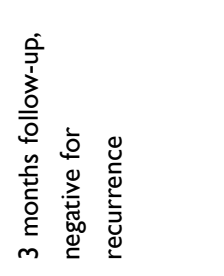 & 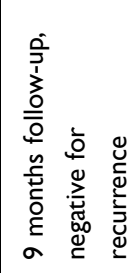 & 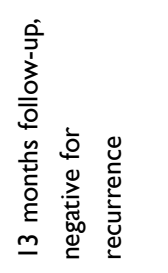 \\
\hline 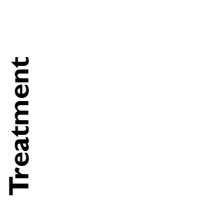 & 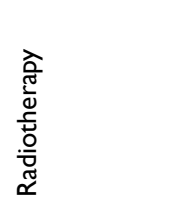 & 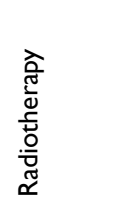 & 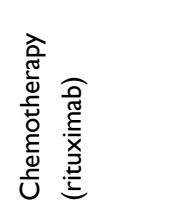 & 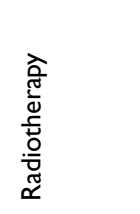 & 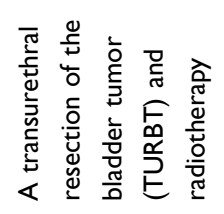 & 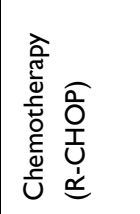 & 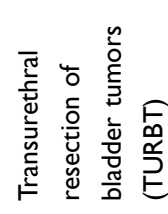 \\
\hline 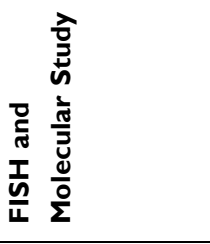 & 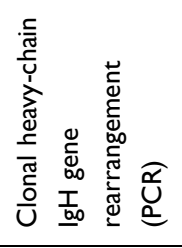 & 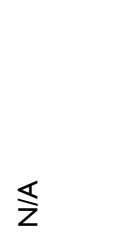 & 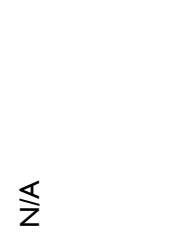 & 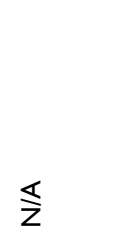 & 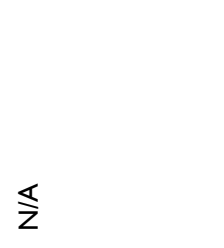 & $\overleftarrow{z}$ & $\stackrel{\nwarrow}{z}$ \\
\hline 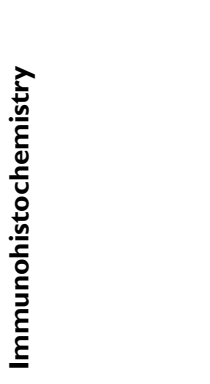 & 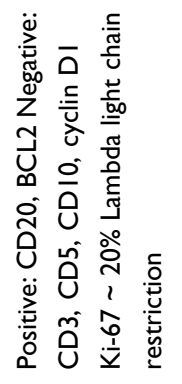 & 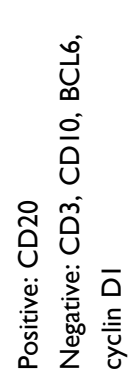 & 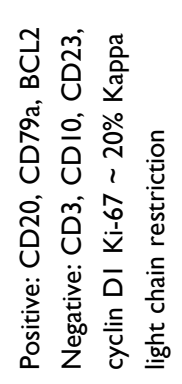 & 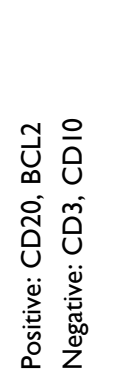 & 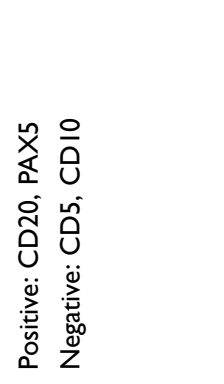 & 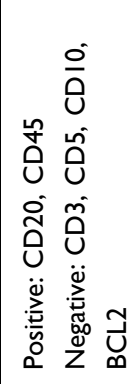 & $\overleftarrow{z}$ \\
\hline ڤ & \llcorner & $\Sigma$ & 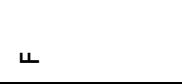 & 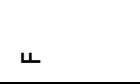 & 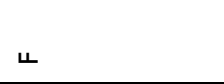 & 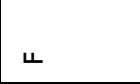 & ч \\
\hline$\stackrel{8}{\&} \underset{0}{\Delta}$ & 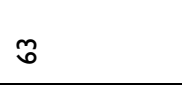 & ஸे & $\stackrel{\infty}{\wedge}$ & $\stackrel{2}{\circ}$ & น & กิ & 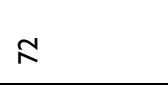 \\
\hline 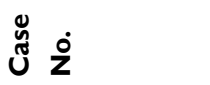 & $\underset{q}{q}$ & in & $\bar{n}$ & กี & $\tilde{n}$ & 苛 & 缉 \\
\hline
\end{tabular}




\begin{tabular}{|c|c|c|c|}
\hline 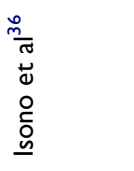 & 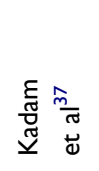 & 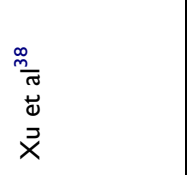 & 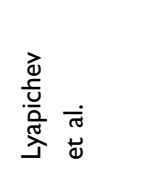 \\
\hline$\frac{\infty}{2}$ & $\frac{\sigma}{i}$ & હ્రి & ڤ్రి \\
\hline 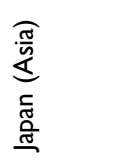 & 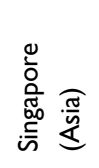 & 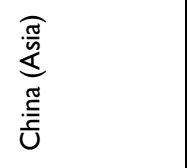 & 芯 \\
\hline 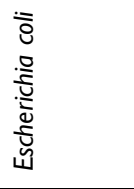 & 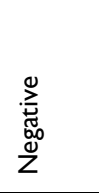 & $\stackrel{\varangle}{z}$ & 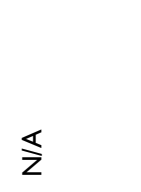 \\
\hline 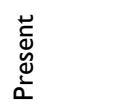 & 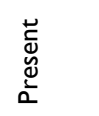 & 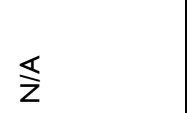 & $\begin{array}{l}\text { 蒿 } \\
\text { 夋 }\end{array}$ \\
\hline 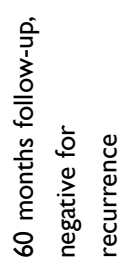 & 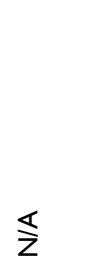 & 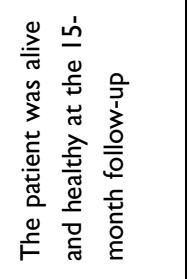 & 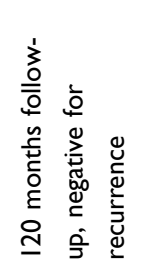 \\
\hline 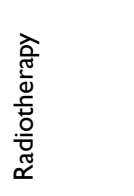 & 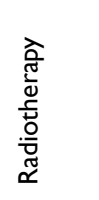 & 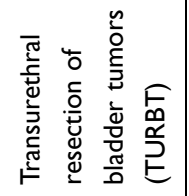 & 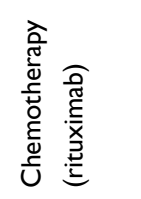 \\
\hline 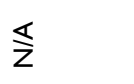 & $\ll$ & 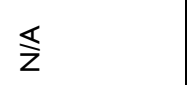 & 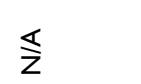 \\
\hline 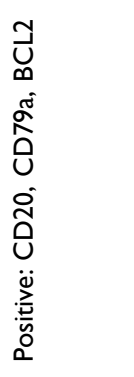 & 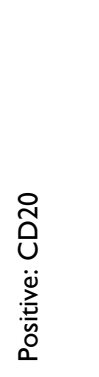 & 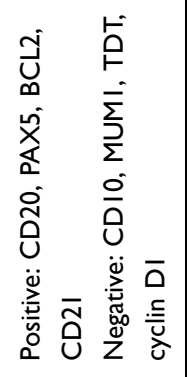 & 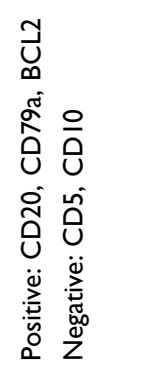 \\
\hline 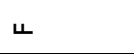 & \llcorner & ч & ч \\
\hline 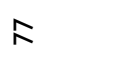 & 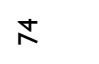 & $\kappa$ & $\stackrel{\infty}{\circ}$ \\
\hline セ̊ & in & $\stackrel{\infty}{\circ}$ & ถ่ \\
\hline
\end{tabular}

with antibiotics. ${ }^{7-9,11,29}$ Eight patients had some surgical procedures with or without consequent radiotherapy and/or chemotherapy. ${ }^{3,16,24,27,31,34,38,45}$ Hughes and colleagues reported one patient who was successfully treated by diathermy. ${ }^{21}$ Majority of the patients (24) were treated with chemotherapy either alone (16) or in combination with radiation (8) (Table 1). Three cases did not have any information about therapeutic approach. ${ }^{17}$ Overall the outcome of the treatment was good with median follow-up time of 64.5 months (from 3 to 156 months).

It is important to acknowledge the limitations of our review. It is possible that some reports did not specify the urinary bladder as an involvement site, so we cannot exclude the possibility of missing a significant number of cases. This is a retrospective study, and patients have not been studied and treated uniformly; therefore, it is impossible to draw a definitive conclusion regarding the pathogenesis of MALT lymphoma of the urinary bladder.

The patient we report has a history of rheumatoid arthritis (RA). The relationship between RA and MALT lymphoma in this patient remains unclear. An increased risk of malignant lymphoma has been reported in patients with RA ${ }^{46,47}$ however, patients with RA usually develop diffuse large B-cell lymphoma. ${ }^{48}$ While MALT lymphoma is common in patients suffering from primary Sjogren syndrome, ${ }^{49}$ MALT lymphoma in patients with RA are exceedingly rare; we identified only 11 single case reports in the literature. ${ }^{50-60}$

\section{Conclusion}

In summary, MALT lymphoma of the urinary bladder is a rare low-grade extranodal B-cell lymphoma which predominantly affects elderly women of Asian origin. The disease often presents with nonspecific symptoms and is strongly associated with cystitis. ${ }^{14,34}$ The prognosis is generally excellent. Tissue biopsy with immunohistochemistry is crucial to reach the final diagnosis. A prominent female predominance, uneven geographic distribution of the cases, dramatic prevalence of cystitis among affected patients, and a success of antibacterial therapy in selected cases suggest the role of urinary tract infection, particularly E. coli, in the pathogenesis of urinary bladder MALT lymphoma.

\section{Ethics Approval and Informed Consent}

The patient reported in the manuscript signed the informed consent/authorization for participation in research (MD Anderson Cancer Center protocol LAB01-473) which 
includes the permission to use data collected in future research projects including presented case details and images used in this manuscript. A copy of the signed consent is kept on file in the patient electronic records.

\section{Disclosure}

The authors declare no conflicts of interest for this work and that there are no conflicts of interest regarding the publication of this article.

\section{References}

1. A clinical evaluation of the International Lymphoma Study Group classification of non-Hodgkin's lymphoma. The non-Hodgkin's lymphoma classification project. Blood. 1997;89(11):3909-3918.

2. Khalil MO, Morton LM, Devesa SS, et al. Incidence of marginal zone lymphoma in the United States, 2001-2009 with a focus on primary anatomic site. Br J Haematol. 2014;165(1):67-77. doi:10.1111/bjh.12730

3. Kuhara H, Tamura Z, Suchi T, Hattori R, Kinukawa T. Primary malignant lymphoma of the urinary bladder. A case report. Acta Pathol Jpn. 1990;40(10):764-769. doi:10.1111/j.1440-1827.1990. tb01541.x

4. Freeman C, Berg JW, Cutler SJ. Occurrence and prognosis of extranodal lymphomas. Cancer. 1972;29(1):252-260.

5. Jitani AK, Mishra J, Sailo SL, Raphael V. Primary urinary bladder mucosa associated lymphoid tissue type lymphoma presenting as a close mimic for genitourinary tuberculosis: case report and review of literature. Urol Ann. 2016;8(1):108-110. doi:10.4103/0974-7796.171491

6. Al-Maghrabi J, Kamel-Reid S, Jewett M, Gospodarowicz M, Wells W, Banerjee D. Primary low-grade B-cell lymphoma of mucosa-associated lymphoid tissue type arising in the urinary bladder: report of 4 cases with molecular genetic analysis. Arch Pathol Lab Med. 2001;125(3):332-336. doi:10.1043/0003-9985(2001) $125<0332:$ PLGBCL $>2.0$. CO;2

7. van den Bosch J, Kropman RF, Blok P, Wijermans PW. Disappearance of a mucosa-associated lymphoid tissue (MALT) lymphoma of the urinary bladder after treatment for Helicobacter pylori. Eur J Haematol. 2002;68(3):187-188. doi:10.1034/j.16000609.2002.01649.x

8. Oscier D, Bramble J, Hodges E, Wright D. Regression of mucosa-associated lymphoid tissue lymphoma of the bladder after antibiotic therapy. J Clin Oncol. 2002;20(3):882. doi:10.1200/ JCO.2002.20.3.882

9. Fujimura M, Chin K, Sekita N, et al. [Regression of mucosa-associated lymphoid tissue lymphoma of the bladder after antibiotic therapy: a case report]. Hinyokika Kiyo. 2008;54 (12):783-786. Japanese.

10. Terada T. Primary CD5-positive mucosa-associated lymphoid tissue lymphoma of the urinary bladder. Ann Diagn Pathol. 2011;15 (5):382-384. doi:10.1016/j.anndiagpath.2011.02.006

11. Krober SM, Aepinus C, Ruck P, Muller-Hermelink HK, Horny HP, Kaiserling E. Extranodal marginal zone B cell lymphoma of MALT type involving the mucosa of both the urinary bladder and stomach. J Clin Pathol. 2002;55(7):554-557. doi:10.1136/jcp.55.7.554

12. Pawade J, Banerjee SS, Harris M, Isaacson P, Wright D. Lymphomas of mucosa-associated lymphoid tissue arising in the urinary bladder. Histopathology. 1993;23(2):147-151. doi:10.1111/j.1365-2559.1993. tb00472.x

13. Fernandez Acenero MJ, Martin Rodilla C, Lopez Garcia-Asenjo J, Coca Menchero S, Sanz Esponera J. Primary malignant lymphoma of the bladder. Report of three cases. Pathol Res Pract. 1996;192 (2):160-165. doi:10.1016/S0344-0338(96)80211-1
14. Kempton CL, Kurtin PJ, Inwards DJ, Wollan P, Bostwick DG. Malignant lymphoma of the bladder: evidence from 36 cases that low-grade lymphoma of the MALT-type is the most common primary bladder lymphoma. Am J Surg Pathol. 1997;21(11):1324-1333. doi:10.1097/00000478-199711000-00007

15. Gallardo J, Gamargo C, Fodor M, Comparini B, Salman P, Yanez M. [MALT lymphoma of the bladder: report of a case]. Rev Med Chil. 1998;126(2):199-201. Spanish.

16. Ando K, Matsuno Y, Kanai Y, et al. Primary low-grade lymphoma of mucosa-associated lymphoid tissue of the urinary bladder: a case report with special reference to the use of ancillary diagnostic studies. Jpn J Clin Oncol. 1999;29(12):636-639. doi:10.1093/jjco/ 29.12.636

17. Bates AW, Norton AJ, Baithun SI. Malignant lymphoma of the urinary bladder: a clinicopathological study of 11 cases. $J$ Clin Pathol. 2000;53(6):458-461. doi:10.1136/jcp.53.6.458

18. Kawakami K, Oka K, Kato M, Shiku H. Whole-bladder irradiation and doxorubicin-containing chemotherapy as successful treatment for a primary mucosa-associated lymphoid tissue lymphoma of the bladder. Int J Hematol. 2000;72(3):346-348.

19. Tasu JP, Geffroy D, Rocher L, et al. Primary malignant lymphoma of the urinary bladder: report of three cases and review of the literature. Eur Radiol. 2000;10(8):1261-1264. doi:10.1007/ s003300000343

20. Wazait HD, Chahal R, Sundurum SK, Rajkumar GN, Wright D, Aslam MM. MALT-type primary lymphoma of the urinary bladder: clinicopathological study of 2 cases and review of the literature. Urol Int. 2001;66(4):220-224. doi:10.1159/000056619

21. Hughes M, Morrison A, Jackson R. Primary bladder lymphoma: management and outcome of 12 patients with a review of the literature. Leuk Lymphoma. 2005;46(6):873-877. doi:10.1080/ 10428190500079829

22. Takahara Y, Kawashima H, Han YS, et al. [Primary mucosa-associated lymphoid tissue (MALT) lymphoma of the urinary bladder]. Hinyokika Kiyo. 2005;51(1):45-48. Japanese.

23. Hatano K, Sato M, Tsujimoto Y, et al. [Primary mucosa-associated lymphoid tissue (MALT) lymphoma of the urinary bladder associated with left renal pelvic carcinoma: a case report]. Hinyokika Kiyo. 2007;53(1):57-60. Japanese.

24. Ueno Y, Sakai H, Tsuruta T, Wajiki M. Mucosa-associated lymphoma of the bladder with relapse in the stomach after successful local treatment. Hinyokika Kiyo. 2007;53(8):575-579.

25. Sen S, Macaulay JH, Allford SL. A case of cerebral arteriovenous malformation in pregnancy associated with MALT lymphoma. $J$ Obstet Gynaecol. 2010;30(3):308-310. doi:10.3109/ 01443610903585234

26. Maninderpal KG, Amir FH, Azad HA, Mun KS. Imaging findings of a primary bladder maltoma. Br J Radiol. 2011;84(1005):e186-e190. doi: $10.1259 / \mathrm{bjr} / 66130737$

27. Szopinski TR, Sudol-Szopinska I, Dzik T, Borowka A, DembowskaBaginska B, Perek D. Incidental sonographic detection of mucosa-associated lymphoid tissue lymphoma of the urinary bladder found in a very young woman: report of a case. $J$ Clin Ultrasound. 2011;39(4):233-235. doi:10.1002/jcu.20786

28. Morita K, Nakamura F, Nannya Y, et al. Primary MALT lymphoma of the urinary bladder in the background of interstitial cystitis. Ann Hematol. 2012;91(9):1505-1506. doi:10.1007/s00277-012-1419-0

29. Lucioni M, Nicola M, Riboni R, et al. Antibiotic therapy-induced remission of bladder mucosa-associated lymphoid tissue (MALT) lymphoma carrying $\mathrm{t}(11 ; 18)(\mathrm{q} 21 ; \mathrm{q} 21)$ apoptosis inhibitor 2-MALT1. $J$ Clin Oncol. 2013;31(19):e304-e306. doi:10.1200/ JCO.2012.46.4800

30. Bacalja J, Ulamec M, Rako D, et al. Persistence of primary MALT lymphoma of the urinary bladder after rituximab with CHOP chemotherapy and radiotherapy. In Vivo (Brooklyn). 2013;27 (4):545-549. 
31. Takahashi H, Shimazaki H, Oda T, et al. Malignant lymphoma case with urinary cytology mimicking that of urothelial carcinoma. Cytopathology. 2013;24(6):412-414. doi:10.1111/cyt.12026

32. Haddad-Lacle JE, Haddad CJ, Villas B. A rare urinary bladder tumour. BMJ Case Rep. 2014;2014:bcr2013202994-bcr2013202994. doi:10.1136/bcr-2013-202994

33. Matsuda I, Zozumi M, Tsuchida YA, et al. Primary extranodal marginal zone lymphoma of mucosa-associated lymphoid tissue type with malakoplakia in the urinary bladder: a case report. Int $\mathrm{J}$ Clin Exp Pathol. 2014;7(8):5280-5284.

34. Vempati P, Knoll MA, Alqatari M, Strauchen J, Malone AK, Bakst RL. MALT lymphoma of the bladder: a case report and review of the literature. Case Rep Hematol. 2015;2015:934374. doi:10.1155/ 2015/934374

35. Hsu JS, Lin CC, Chen YT, Lee YC. Primary mucosa-associated lymphoid tissue lymphoma of the urinary bladder. Kaohsiung J Med Sci. 2015;31(7):388-389. doi:10.1016/j.kjms.2015.04.001

36. Isono M, Sato A, Kimura F, Asano T. A case of mucosa-associated lymphoid tissue lymphoma of the bladder successfully treated with radiotherapy. Urol Case Rep. 2018;16:1-3. doi:10.1016/j. eucr.2017.09.011

37. Kadam PD, Han HC, Kwok JL. An uncommon case of mucosa-associated lymphoid tissue (MALT) tumor of the bladder. Int Urogynecol J. 2019;30(6):1017-1018. doi:10.1007/s00192-0183813-1

38. Xu H, Chen Z, Shen B, Wei Z. Primary bladder mucosa-associated lymphoid tissue lymphoma: a case report and literature review. Medicine (Baltimore). 2020;99(28):e20825. doi:10.1097/ MD.0000000000020825

39. Chen YR, Hung LY, Chang KC. Mucosa-associated lymphoid tissue-type lymphoma presenting as a urethral caruncle with urinary bladder involvement. Int $J$ Urol. 2014;21(10):1073-1074. doi:10.1111/iju.12507

40. Yuille FA, Angus B, Roberts JT, Vadanan BS. Low grade MALT lymphoma of the urinary bladder. Clin Oncol ( $R$ Coll Radiol). 1998;10(4):265-266. doi:10.1016/S0936-6555(98)80016-2

41. Kakuta Y, Katoh T, Saitoh J, Yazawa K, Hosomi M, Itoh K. [A case of primary mucosa-associated lymphoid tissue lymphoma of the bladder regressed after rituximab in combination with $\mathrm{CHOP}$ chemotherapy]. Hinyokika Kiyo. 2006;52(12):951-954. Japanese.

42. Terasaki Y, Okumura H, Ishiura Y, et al. [Primary mucosa-associated lymphoid tissue lymphoma of the urinary bladder successfully treated by radiotherapy and rituximab]. Rinsho Ketsueki. 2008;49(1):30-34. Japanese.

43. Painemal Duarte C, Gallardo J, Valdebenito JP, Gamargo C, Rubio B, Harbst H. [MALT lymphoma of the bladder. Report of a case]. Arch Esp Urol. 2001;54(10):1138-1140. Spanish.

44. Mizuno K, Nakanishi S, Sakatani T, et al. [A case of primary mucosa-associated lymphoid tissue-type lymphoma of the urinary bladder that progressed after antibiotic therapy]. Hinyokika Kiyo. 2013;59(4):239-242. Japanese.

45. Ozawa M, Suenaga S, Ishii T, Suzuki H, Tsuchiya N, Ohtake H. [Primary malignant lymphoma of the bladder diagnosed by transurethral bladder tumor resection: a case report]. Nihon Hinyokika Gakkai Zasshi. 2018;109(1):45-49. Japanese. doi:10.5980/ jpnjurol.109.45

46. Baecklund E, Askling J, Rosenquist R, Ekbom A, Klareskog L. Rheumatoid arthritis and malignant lymphomas. Curr Opin Rheumatol. 2004;16(3):254-261. doi:10.1097/00002281-20040500000014
47. Zintzaras E, Voulgarelis M, Moutsopoulos HM. The risk of lymphoma development in autoimmune diseases: a meta-analysis. Arch Intern Med. 2005;165(20):2337-2344. doi:10.1001/ archinte.165.20.2337

48. Baecklund E, Sundstrom C, Ekbom A, et al. Lymphoma subtypes in patients with rheumatoid arthritis: increased proportion of diffuse large B cell lymphoma. Arthritis Rheum. 2003;48(6):1543-1550. doi:10.1002/art.11144

49. Kauppi M, Pukkala E, Isomaki H. Elevated incidence of hematologic malignancies in patients with Sjogren's syndrome compared with patients with rheumatoid arthritis (Finland). Cancer Causes Control. 1997;8(2):201-204. doi:10.1023/A:1018472213872

50. Magnoli F, Cimetti L, Bernasconi B, et al. Primary extranodal marginal cell lymphoma, MALT type, of the endometrium arising in a patient with rheumatoid arthritis: report of a case. Int $J$ Gynecol Pathol. 2016;35(4):327-332. doi:10.1097/PGP.0000000000000244

51. Kobayashi Y, Kimura K, Fujitsu Y, Shinkawa K, Muta H, Sonoda KH. Methotrexate-associated orbital lymphoproliferative disorder in a patient with rheumatoid arthritis: a case report. Jpn J Ophthalmol. 2016;60(3):212-218. doi:10.1007/s10384-016-0439-z

52. Gonzalez-Murillo EA, Castro-Rodriguez A, Sanchez-Venegas JC, Pena-Ruelas CI. Subglottic MALT lymphoma of the larynx in a patient with rheumatoid arthritis. Acta Otorrinolaringol Esp. 2014;65(5):317-319. doi:10.1016/j.otoeng.2013.03.001

53. Go H, Cho HJ, Paik JH, et al. Thymic extranodal marginal zone B-cell lymphoma of mucosa-associated lymphoid tissue: a clinicopathological and genetic analysis of six cases. Leuk Lymphoma. 2011;52(12):2276-2283. doi:10.3109/ 10428194.2011.596968

54. Mikolaenko I, Listinsky CM. Systemic CD5+ MALT lymphoma: presentation with Waldenstrom syndrome. Ann Diagn Pathol. 2009;13(4):272-277. doi:10.1016/j.anndiagpath.2008.04.010

55. Sordet C, Mrabet D, Ardizzone M, Marcellin L, Hirschorn P, Sibilia J. A centrofacial B lymphoma in a rheumatoid arthritis patient. Eur J Intern Med. 2007;18(1):71-73. doi:10.1016/j. ejim.2006.04.019

56. Douglas KM, Raza K, Stevens R, Erb N, Jones EL, Kitas GD. Bronchial MALT lymphoma in longstanding rheumatoid arthritis. Rheumatology (Oxford). 2005;44(5):687-689. doi:10.1093/rheumatology/keh545

57. Kim JM. Primary extranodal marginal zone B-cell lymphoma of mucosa-associated lymphoid tissue-type in the thymus of a patient with Sjogren's syndrome and rheumatoid arthritis. J Korean Med Sci. 2003;18(6):897-900. doi:10.3346/jkms.2003.18.6.897

58. Sutcliffe N, Smith C, Speight PM, Isenberg DA. Mucosa-associated lymphoid tissue lymphomas in two patients with rheumatoid arthritis on second-line agents, and secondary Sjogren's syndrome. Rheumatology (Oxford). 2000;39(2):185-188. doi:10.1093/rheumatology/39.2.185

59. Yokose T, Kodama T, Matsuno Y, Shimosato Y, Nishimura M, Mukai K. Low-grade B cell lymphoma of mucosa-associated lymphoid tissue in the thymus of a patient with rheumatoid arthritis. Pathol Int. 1998;48(1):74-81. doi:10.1111/j.1440-1827.1998. tb03832.x

60. Serefhanoglu S, Tapan U, Ertenli I, Kalyoncu U, Uner A. Primary thyroid marginal zone B-cell lymphoma MALT-type in a patient with rheumatoid arthritis. Med Oncol. 2010;27(3):826-832. doi:10.1007/ s12032-009-9293-X 


\section{Publish your work in this journal}

Research and Reports in Urology is an international, peer-reviewed, open access journal publishing original research, reports, editorials, reviews and commentaries on all aspects of adult and pediatric urology in the clinic and laboratory including the following topics: Pathology, pathophysiology of urological disease; Investigation and treatment of urological disease; Pharmacology of drugs used for the treatment of urological disease. The manuscript management system is completely online and includes a very quick and fair peer-review system, which is all easy to use. Visit http://www.dovepress.com/ testimonials.php to read real quotes from published authors. 